\title{
FUNCIONALIZACIÓN DE TEXTILES DE ALGODÓN CON NANOPARTÍICULAS DE $\mathrm{ZnO}_{2}$
}

\author{
Carmen Uribe Valenzuela ${ }^{a}$, Elsa Roca Meneses ${ }^{\mathrm{a}}$, Marco Brañez Sánchez ${ }^{\mathrm{a}}$, \\ Jessica Álvarez Alayo ${ }^{a}$, L. Esmeralda Román Mendoza a,b, Dora Maurtua Torres ${ }^{\mathrm{c}}$, \\ José L. Solís Veliz ${ }^{\mathrm{d}}$ y Mónica M. Gómez León ${ }^{* d}$
}

\begin{abstract}
RESUMEN
Se sintetizó nanopartículas de peróxido de zinc $\left(\mathrm{ZnO}_{2}\right)$ por el método sol-gel y se las caracterizó estructural y morfológicamente. Las nanopartículas sintetizadas fueron suspendidas en un medio acuoso para funcionalizar tejidos textiles de algodón, obteniendo textiles con propiedades antimicrobianas. La funcionalización fue mediante el proceso de impregnación sobre tejido seco, haciendo uso del equipo denominado Foulard de impregnación. Las muestras textiles impregnadas fueron analizadas microbiológicamente frente a cepas de bacterias Pseudomonas aeruginosa (ATCC 10145) y Escherichia coli (ATCC 25922), posterior a estos análisis se realizaron controles de solideces, análisis de sus propiedades físicas y control del color. De los análisis realizados se concluye que los textiles funcionalizados con nanopartículas de $\mathrm{ZnO}_{2}$ poseen propiedades antimicrobianas. Además las propiedades físicas y las solideces del textil no son afectadas luego del proceso de funcionalización.
\end{abstract}

Palabras clave: Nanopartículas, funcionalización, textiles antimicrobianos, control de calidad textil, análisis microbiológico.

\section{FUNCIONALIZATION OF COTTON TEXTILES WITH ZnO2 NANOPARTICLES}

\begin{abstract}
Zinc peroxide $\left(\mathrm{ZnO}_{2}\right)$ nanoparticles were synthesized by sol-gel method and characterized structurally and morphologically. The synthesized nanoparticles were suspended in aqueous medium to functionalize cotton textile fabrics, getting textiles with antimicrobial properties.

a. Facultad de Ingeniería Química y Textil, Universidad Nacional de Ingeniería, Av. Túpac Amaru 210, Lima 25, Perú

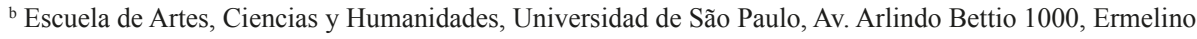
Matarazzo, São Paulo, Brasil.

c Universidad Peruana Cayetano Heredia, Facultad de Ciencias y Filosofía, Av. Honorio Delgado 430, Lima 31, Perú

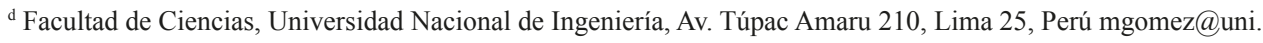
edu.pe
\end{abstract}


The functionalization was by impregnation process over dry woven, making use of equipment called impregnation Padder. The impregnated textile samples were microbiologically analyzed against Pseudomonas aeruginosa (ATCC 10145) and Escherichia coli (ATCC 25922) strains. Subsequently, fastness control, physical properties studies and color control analysis were performed over the textiles. We concluded that the textiles functionalized with $\mathrm{ZnO} 2$ nanoparticles have antimicrobial properties, moreover, physical properties and textile fastness are not affected after the functionalization process.

Key words: Nanoparticles, functionalization, antimicrobial textile, textile quality control, microbiological analysis.

\section{INTRODUCCIÓN}

Actualmente, en la industria textil se viene investigando y desarrollando productos con propiedades diferentes a los que normalmente tienen, estos nuevos productos son denominados textiles polifuncionales o textiles inteligentes ${ }^{1-5}$, podemos mencionar a los textiles que tienen propiedades ignífugas ${ }^{6-8}$, de protección de rayos $\mathrm{UV}^{6,9}$, antimicrobianos ${ }^{10-14} \mathrm{e}$ incluso otros que funcionan como biosensores. ${ }^{15-17}$ Los nuevos acabados textiles con propiedades específicas no afectan la calidad estética del textil, tal es el caso de los acabados antimicrobianos a base de nanomateriales. ${ }^{2,18-20}$ Entre los nanomateriales más prometedores con propiedades antimicrobianas y antivirales son las nanopartículas metálicas de $\mathrm{Ag},{ }^{21} \mathrm{Cu},{ }^{22} \mathrm{Au},{ }^{23} \mathrm{Zn},{ }^{24}{ }^{25}$ entre otros, que presentan aumento de su actividad química debido a la gran relación de superficie a volumen y la estructura de la superficie cristalográfica. ${ }^{26}$

El uso de nanopartículas de peróxido de zinc $\left(\mathrm{ZnO}_{2}\right)$ se ha visto como un medio eficaz para detener enfermedades infecciosas de la piel e infecciones de heridas, ello debido a las propiedades antibacterianas y antifúngicas que posee. ${ }^{27-32}$ En el presente artículo reportamos la síntesis y caracterización de nanopartículas $\mathrm{ZnO}_{2}$, así como los resultados obtenidos del control de solideces, control de color, análisis microbiológicos y pruebas físicas de los textiles funcionalizados con nanopartículas de $\mathrm{ZnO}_{2}$. Las evaluaciones de los textiles se realizaron empleando normas técnicas estándares internacionales, así como también técnicas de caracterización como microscopía electrónica para los estudios morfológicos.

\section{PARTE EXPERIMENTAL}

En la parte experimental de la investigación se realizó la síntesis de las nanopartículas de $\mathrm{ZnO}_{2}$ y su caracterización por técnicas estructurales y morfológicas. Luego, estas nanopartículas fueron empleadas para la funcionalización de los materiales textiles.

Las nanopartículas de $\mathrm{ZnO}_{2}$ fueron obtenidas por el método sol-gel ${ }^{33}$ partiendo de una solución de acetato de zinc y peróxido de hidrógeno bajo radiación $\mathrm{UV}^{28} \mathrm{El}$ esquema de síntesis de nanopartículas de $\mathrm{ZnO}_{2}$ se muestra en la figura 1 y la reacción que se postula para el proceso de síntesis es: ${ }^{34,35}$ 


$$
\mathrm{Zn}\left(\mathrm{CH}_{3} \mathrm{COO}\right)_{2}+2 \mathrm{H}_{2} \mathrm{O}_{(\mathrm{ac})}+\mathrm{H}_{2} \mathrm{O}_{2} \stackrel{\mathrm{UV}}{\rightarrow} \mathrm{ZnO}_{2} \downarrow+2\left(\mathrm{CH}_{3} \mathrm{COOH}\right)_{(\mathrm{ac})}+2 \mathrm{H}_{2} \mathrm{O}
$$

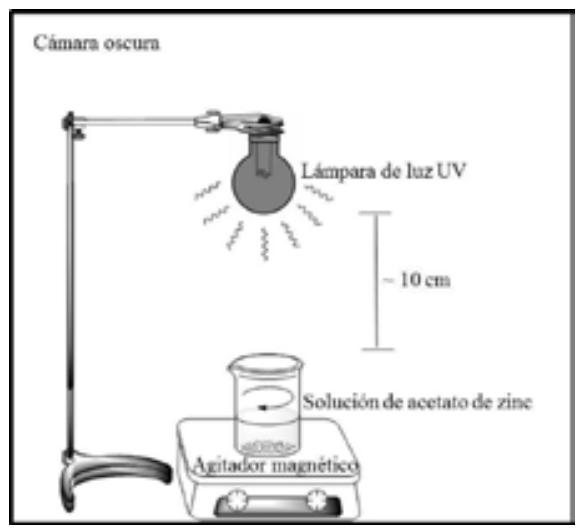

Figura 1. Esquema de la síntesis de nanopartículas de $\mathrm{ZnO} 2$ por radiación UV.

\section{RESULTADOS Y DISCUSIÓN}

A continuación se presentan los resultados experimentales de la caracterización estructural y morfológica de las nanopartículas de $\mathrm{ZnO}_{2}$, así como los resultados de la evaluación de calidad y microbiológico de los textiles funcionalizados con dichas nanopartículas.

\section{Difracción de Rayos X}

La caracterización estructural de las nanopartículas de $\mathrm{ZnO}_{2}$ se realizó empleando el método de difracción de rayos X, para ello se empleó el difractómetro Rigaku Miniflex II Desktop, con fuente de radiación de $\mathrm{CuK} \alpha 1(\lambda=0.15045 \mathrm{~nm})$ a $30 \mathrm{kV}, 20 \mathrm{~mA}$ y velocidad de barrido de $3^{\circ} / \mathrm{min}$. La figura 2 muestra el difractograma obtenido del polvo de $\mathrm{ZnO}_{2}$. Se pueden observar 4 picos para los ángulos $31,7^{\circ}, 36,8^{\circ}, 53,1^{\circ}$ y $63,2^{\circ}$ asignados a las reflexiones [111], [200], [220] y [311] del correspondientes a la estructura cúbica del $\mathrm{ZnO}_{2},{ }^{34}$, respectivamente. El tamaño del dominio cristalino fue calculado empleando la Fórmula de Scherrer ${ }^{36}$ la cual arrojó un valor de $4 \mathrm{~nm}$.

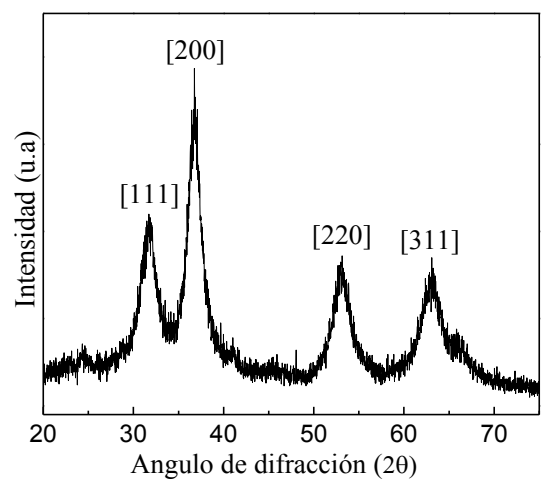

Figura 2. Difractograma de rayos $\mathrm{X}$ de las nanopartículas $\mathrm{ZnO}_{2}$. 


\section{Microscopía electrónica de barrido de emisión de campo}

La caracterización morfológica de las nanopartículas de $\mathrm{ZnO}_{2}$ se realizó por microscopía electrónica de barrido de emisión de campo utilizando el microscopio electrónico JEOL JSM$7401 \mathrm{~F}$ operado a $5 \mathrm{kV}$. La figura 3 muestra las micrografías electrónicas de las nanopartículas de $\mathrm{ZnO}_{2}$, se pueden observar conglomerados esféricos de tamaños de diámetro entre $\sim 80$ y $200 \mathrm{~nm}$ los cuales están conformados por dominios cristalinos de tamaños muchos más pequeños que no se pueden identificar en la imagen.

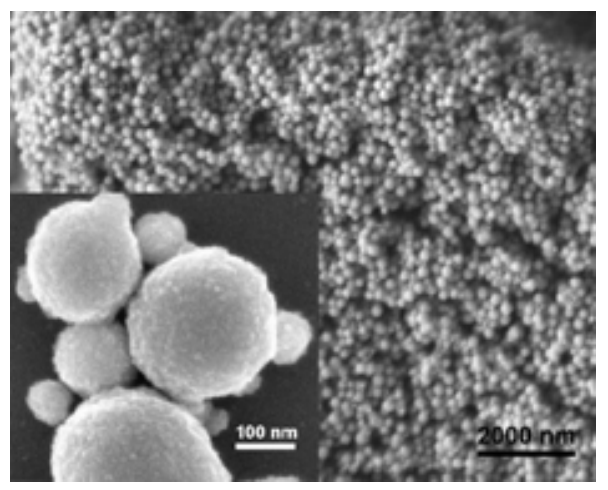

Figura 3. Micrografías electrónicas de barrido de emisión de campo de las nanopartículas $\mathrm{ZnO}_{2}$.

\section{Espectroscopía infrarroja con transformada de Fourier}

Los análisis de espectroscopía de infrarroja de las nanopartículas de $\mathrm{ZnO}_{2}$ se llevaron a cabo en un espectrofotómetro IR Prestige-21 Shimadzu en el rango de 4000 a $400 \mathrm{~cm}^{-1}$, empleando pastillas de bromuro de potasio $(\mathrm{KBr})$ que contuvieron las nanopartículas y usando como referencia el $\mathrm{KBr}$ puro.

En la figura 4 se observa el espectro de transmisión, en el cual los picos corresponden a procesos de absorción. La banda que se presenta claramente en el rango de $3400 \mathrm{~cm}^{-1}$ a $3600 \mathrm{~cm}-$ 1 se atribuye a la absorción del modo stretching del enlace $\mathrm{O}-\mathrm{H}$ - que puede provenir de las moléculas de agua adsorbidas superficialmente a las nanopartículas de $\mathrm{ZnO}_{2}{ }^{34,}{ }^{37}$ Alrededor de $3000 \mathrm{~cm}^{-1}$ se observan pequeños picos característicos al modo stretching del enlace $\mathrm{C}-\mathrm{H}$ del grupo metilo $\left(-\mathrm{CH}_{3}\right){ }^{34}$ Entre $1250 \mathrm{~cm}^{-1}$ y $1750 \mathrm{~cm}^{-1}$ los picos formados corresponden a los modos de vibración del acetato de zinc $^{-38}$ lo que podría indicar que permaneció algo de reactante sin reaccionar. La banda a longitudes de onda menores a $500 \mathrm{~cm}^{-1}$ se atribuye al modo de vibración stretching del enlace Zn-O. ${ }^{39}, 40$ 


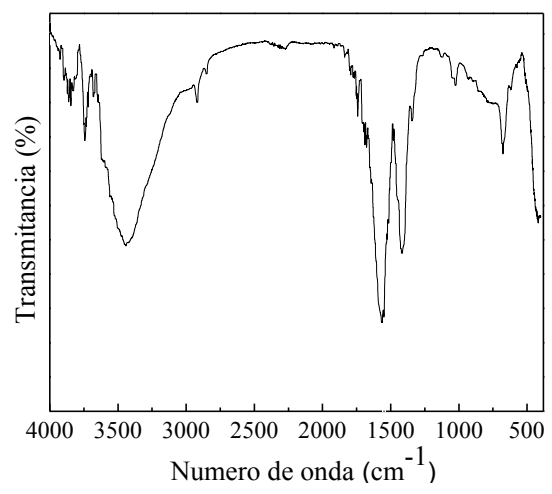

Figura 4. Espectros de infrarrojo con transformada de Fourier de nanopartículas $\mathrm{ZnO}_{2}$.

\section{Funcionalización del textil con nanopartículas de $\mathrm{ZnO}_{2}$}

Para la funcionalización de los textiles con nanopartículas de $\mathrm{ZnO}_{2}$ se utilizaron suspensiones coloidales a 20, 40 y $60 \mathrm{~g} / 1$, donde el medio líquido fue agua destilada y alcohol isotridecílico al $2 \%$.

El proceso de funcionalización se realizó por el método de impregnación en seco, haciendo uso del Foulard de impregnación. El proceso consistió en pasar el textil a través de la suspensión coloidal, contenida en un recipiente, para luego ser exprimido entre dos rodillos que se encontraron a una presión de 4 bar, todo ello con la finalidad de lograr una absorción uniforme y controlada a lo largo y ancho del textil. Este proceso suele expresarse en forma de porcentaje (\%) de absorción, siempre con relación al peso seco del textil, por lo cual es indispensable pesar el textil antes y después del proceso de impregnación. Este \% de absorción es denominado pick up y se expresa como la cantidad de producto aplicado a la tela en función a la base seca de esta y se calcula según la siguiente ecuación:

$$
\frac{\text { Peso }_{\text {húmedo }}-\text { Peso }_{\text {seco }}}{\text { Peso }_{\text {seco }}} \times 100
$$

El pick up obtenido normalmente durante este trabajo se encontró entre 70 y $80 \%$. Posteriormente para eliminar el agua remanente en el textil fue llevado a una estufa para ser secado a una temperatura de $120^{\circ} \mathrm{C}$ por un tiempo de $3 \mathrm{~min}$.

\section{Control de calidad}

En este trabajo se realizaron las pruebas físicas, controles de solideces y control de color del textil no funcionalizado (TNF) y a los textiles funcionalizados con suspensiones de $20 \mathrm{~g} / 1$ (TF20), 40 g/l (TFs40) y $60 \mathrm{~g} / \mathrm{l}$ (TF60) de nanopartículas de $\mathrm{ZnO}_{2}$.

\section{Pruebas físicas}

La determinación de las pruebas físicas de un textil consiste en especificar completamente las características mecánicas que este posee. En el presente trabajo se desarrollaron las siguientes 
evaluaciones del tejido: ligamento, densidad, gramaje, resistencia y título del hilo que lo forma. Todas estas evaluaciones se realizaron siguiendo normas técnicas internacionales.

\section{Ligamento del tejido}

El ligamento de un tejido es la manera de entrecruzar los hilos (urdimbre) con las pasadas (trama) y cuyo conjunto de evoluciones se repite constantemente a lo largo y ancho del tejido. Los ligamentos fundamentales son: tafetán, sarga y raso. En el presente trabajo el ligamento se determinó con la norma NTP 231.141:1985: 2010. En la figura 5, se observa claramente que el ligamento de los textiles empleados es una sarga 3/1, lo que significa que la evolución del hilo es 3 tomados (hilo encima de la pasada) y 1 dejado (hilo debajo de la pasada).
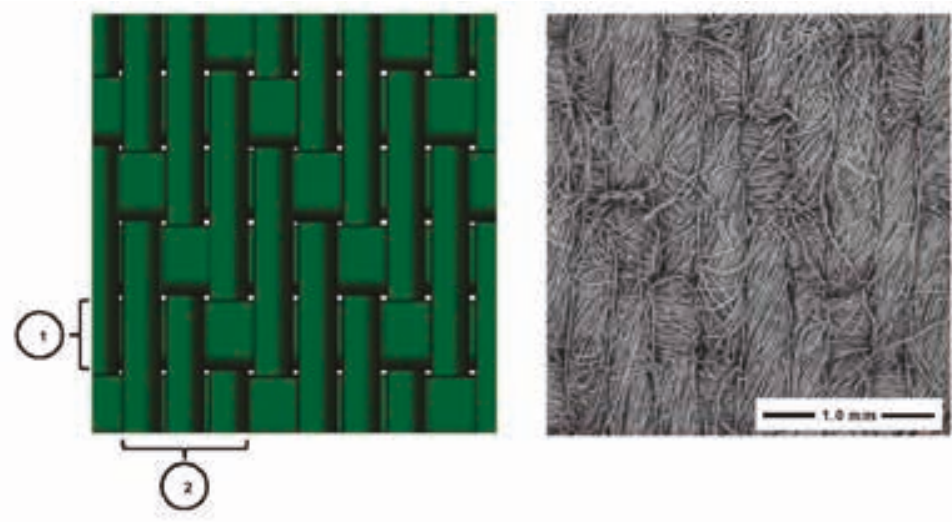

Figura 5. (a) Esquema del ligamento sarga $3 / 1$ en donde (1) hilos de trama y (2) hilos de urdimbre. (b) Micrografía electrónica de barrido del tejido utilizado en el presente trabajo

\section{Densidad del tejido}

Es la cantidad de hilos por unidad de longitud del tejido. El proceso para su determinación consistió en contar el número de hilos que están dentro de una pulgada lineal, haciendo uso del instrumento denominado Cuentahílos. La densidad del tejido fue determinada con la norma ASTM D3775:2012.

La tabla 1 muestra los resultados para los textiles funcionalizados con suspensiones de diferentes concentraciones y del textil no funcionalizados (TNF). Claramente se observa que la cantidad de hilos en el sentido de la urdimbre permanece constante en todas las muestras de tela, mientras que en el sentido de los hilos de trama hay un incremento de la cantidad de hilos (encogimiento en esa dirección) para los textiles funcionalizados. Este ligero encogimiento observado es una propiedad típica del algodón, por lo que no podemos con certeza atribuirlo al proceso de funcionalización y al mecanismo que podrían estar jugando las nanopartículas en él. 
Tabla 1. Resultados de la densidad del tejido de los textiles funcionalizados con suspensiones de diferentes concentraciones de nanopartículas, y del textil no funcionalizado (TNF).

\begin{tabular}{ccc}
\hline \multirow{2}{*}{ Muestras textiles } & \multicolumn{2}{c}{$\mathrm{N}^{\circ}$ Hilos/pulgada } \\
\cline { 2 - 3 } & Urdimbre & Trama \\
\hline TNF & 116 & 57 \\
TF20 & 116 & 59 \\
TF40 & 116 & 59 \\
TF60 & 116 & 60 \\
\hline
\end{tabular}

\section{Gramaje del tejido}

Este parámetro da información de la cantidad de masa textil por unidad de área $\left(\mathrm{g} / \mathrm{m}^{2}\right)$. Para su determinación, se usó el instrumento denominado Sacabocado, que corta el tejido en forma circular con un área de $100 \mathrm{~cm}^{2}$. El gramaje del tejido fue determinado haciendo uso de la norma ASTM D3776 - 09a:2013.

La tabla 2 presenta los resultados obtenidos para las muestras textiles analizadas. Se observa que, el gramaje de las muestras se incrementa con el aumento de la concentración de la suspensión de nanopartículas de $\mathrm{ZnO}_{2}$. Este resultado podríamos fácilmente esperarlo considerando que al aumentar la concentración de nanopartículas de $\mathrm{ZnO}_{2}$ en la suspensión, aumenta la presencia de ellas en el textil y por lo tanto la masa de este se incrementa.

Tabla 2. Resultados del gramaje de los textiles funcionalizados con suspensiones de diferentes concentraciones de nanopartículas, y del textil no funcionalizado

(TNF).

\begin{tabular}{cc}
\hline Muestras textiles & Gramaje $\left(\mathrm{g} / \mathrm{m}^{2}\right)$ \\
\hline TNF & 265.87 \\
TF20 & 272.93 \\
TF40 & 275.00 \\
TF60 & 280.99 \\
\hline
\end{tabular}

\section{Resistencia del tejido}

Este parámetro representa la carga en libras o kilogramos que soporta el textil por unidad de longitud, para la evaluación de la resistencia a la rotura de los tejidos se utilizó el Método Grab y fue determinado con la norma ASTM D5034 - 09:2013.

Los resultados de resistencia de los tejidos analizados se muestran en la tabla 3, donde se observa que en el sentido de la urdimbre, la relación resistencia/cantidad de nanopartículas es inversa. Así, la muestra textil funcionalizada con una suspensión de concentración igual a 60 $\mathrm{g} / \mathrm{l}$ tiene la menor resistencia. En el sentido de la trama se tiene un aumento de la resistencia 
para todos los textiles funcionalizados. Este efecto podemos interpretarlo en conexión con los resultados obtenidos para la densidad del tejido, donde hay aumento de hilos de trama, lo que le conferiría mayor resistencia en esa dirección.

Por otro lado, en dirección de los hilos de urdimbre se observa que el textil funcionalizado con la suspensión de concentración $20 \mathrm{~g} / 1$ presenta un aumento de la resistencia. Pero este valor disminuye sostenidamente a medida que aumenta la presencia de nanopartículas en el textil, esto podría deberse a que el $\mathrm{ZnO} 2$ posiblemente afecte químicamente a la fibra.

Tabla 3. Resultados de la resistencia de los textiles funcionalizados con suspensiones de diferentes concentraciones de nanopartículas, y del textil no funcionalizado (TNF).

\begin{tabular}{ccc}
\hline \multirow{2}{*}{ Muestras textiles } & \multicolumn{2}{c}{ Unidades $(\mathrm{kg} / \mathrm{m})$} \\
\cline { 2 - 3 } & Urdimbre & Trama \\
\hline TNF & 745.41 & 608.92 \\
TF20 & 813.65 & 673.23 \\
TF40 & 801.84 & 666.67 \\
TF60 & 633.86 & 717.85 \\
\hline
\end{tabular}

\section{Título del hilo}

Es una característica técnica que relaciona la masa por unidad de longitud, o viceversa, del hilo. Para su determinación, en este trabajo se empleó la norma ASTM D1059 - 01:2010 haciendo uso del sistema inglés $(\mathrm{Ne}){ }^{41}$

La tabla 4 muestra los resultados de título del hilo de las muestras textiles analizadas donde se observa una mínima diferencia entre los títulos de los hilos de urdimbre de todas las muestras, aproximadamente entre los valores de 0.04 y 0.14 . El título de los hilos de trama tiene la tendencia a disminuir conforme se incrementa la concentración de suspensión de $\mathrm{ZnO}_{2}$ impregnada al tejido textil. La leve disminución del título de los hilos de trama podría deberse al incremento de la presencia de las nanopartículas de $\mathrm{ZnO}_{2}$, ya que estas al adherirse en la superficie de los hilos hacen que aumenten el peso y por tanto que el título sea más grueso.

Tabla 4. Título del hilo en sistema inglés (Ne) para los textiles funcionalizados con suspensiones de diferentes concentraciones de nanopartículas, y del textil no funcionalizado (TNF).

\begin{tabular}{ccc}
\hline \multirow{2}{*}{ Muestras textiles } & \multicolumn{2}{c}{$\mathrm{Ne}$} \\
\cline { 2 - 3 } & Urdimbre & Trama \\
\hline TNF & 19.29 & 11.82 \\
TF20 & 19.38 & 11.62 \\
TF40 & 19.25 & 11.43 \\
TF60 & 19.11 & 11.42 \\
\hline
\end{tabular}




\section{Solideces}

La solidez es la resistencia que presenta un textil teñido a variar o perder su color al ser sometido a la acción de un determinado agente, pudiendo dar lugar a la degradación del color y/o a la descarga sobre otros textiles. El agente es la causa capaz de producir la modificación visual del color en los textiles teñidos, estampados o con acabados específicos.

En el presente trabajo las solideces se evaluaron desarrollando normas técnicas para determinar la respuesta del textil al lavado, al frote y al sudor. La evaluación de solideces, se realizó en la cabina de luces con la luz D65 incidiendo en ángulo de $45^{\circ}$, además de emplear escalas de grises estandarizadas por la Asociación Americana de Químicos y Coloristas Textiles (AATCC del término en inglés American Association of Textile Chemists and Colorists), las cuales son: Escala de Grises para Cambio de Color (EGCC) y Escala de Grises para Transferencia de Color (EGTC), estas valoraciones muestran el cambio y transferencia del color luego que el material textil es sometido a una evaluación de solidez, la valoración se da según los grados 5, 4-5, 4, 3-4, 3, 2-3, 2, 1-2, 1, donde el grado 5 es el de mayor solidez (no hay cambio o transferencia de color) y 1 el de más baja solidez (hay un gran cambio o una gran transferencia de color).

\section{Solidez al lavado}

Determinada con la norma ISO 105/C06:2006, que consiste en lavar con una solución de detergente sin blanqueador óptico los tejidos textiles cosidos a un testigo multifibra en presencia de billas de acero, todo ello dentro de vasos de acero que giran en los ejes de la máquina denominada Launderómetro por un período de $45 \mathrm{~min}$.

La tabla 5 muestra los resultados obtenidos luego de la evaluación de la solidez al lavado, donde se puede observar que el TNF y los textiles funcionalizados con diferentes concentraciones de suspensiones de nanopartículas de $\mathrm{ZnO} 2$ presentan el mismo grado de valoración en transferencia de color sobre el testigo de multifibra. Con respecto al cambio de color, se observa que el TNF y los textiles funcionalizados con suspensiones de nanopartículas con concentraciones de $20 \mathrm{~g} / 1$ y $40 \mathrm{~g} / 1$ no presentan cambio de color notorio (grado $4-5,5$ ), mientras el textil funcionalizado con una concentración de $60 \mathrm{~g} / \mathrm{l}$, presenta un cambio de color moderado (grado 3), esto se justifica por las evaluaciones visuales realizadas luego de la funcionalización de los textiles, donde a mayor concentración el textil se torna más claro.

Tabla 5. Resultados de la evaluación de solidez al lavado para los textiles funcionalizados con suspensiones de diferentes concentraciones de nanopartículas, y del textil no funcionalizado (TNF) según Norma ISO 105/C06:2006.

\begin{tabular}{clcccc}
\hline \multirow{2}{*}{ Evaluación } & \multicolumn{2}{c}{ Testigo multifibra } & \multicolumn{4}{c}{ Muestras textiles } \\
\cline { 3 - 6 } & \multicolumn{1}{c}{ DW } & TNF & TF20 & TF40 & TF60 \\
\hline \multirow{5}{*}{ Transferencia de } & Di-acetato & $4-5$ & $4-5$ & $4-5$ & $4-5$ \\
color & Algodón blanqueado & $4-5$ & 4 & 4 & 4 \\
& Poliamida & 4 & $4-5$ & 4 & $4-5$ \\
& Poliéster & $4-5$ & $4-5$ & $4-5$ & $4-5$ \\
& Acrílico & 5 & 5 & $4-5$ & $4-5$ \\
& Lana & $4-5$ & 4 & $4-5$ & 4 \\
\hline Cambio de color & & $4-5$ & $4-5$ & $4-5$ & 3 \\
\hline
\end{tabular}




\section{Solidez al frote}

La solidez al frote fue determinada con la norma AATCC 8:2013, que consiste en sujetar con un dispositivo de sujeción el textil a evaluar en la base del equipo denominado Frotómetro que posee una lija de agua, de tal manera que la dirección diagonal del textil se encuentre en dirección paralela al movimiento de la clavija del equipo. En la Tabla 6 se presentan los datos para los textiles evaluados. Se observa que la concentración de nanopartículas interfiere mínimamente en la solidez al frote en seco. Respecto al frote húmedo, los textiles funcionalizados y el TNF presentan el mismo resultado, no importando la concentración de la suspensión de nanopartículas aplicada, esto debido a que la funcionalización a diferentes concentraciones afecta mínimamente y del mismo modo al tejido textil.

Tabla 6. Resultados de la evaluación de solidez al frote para los textiles funcionalizados con suspensiones de diferentes concentraciones y del textil no funcionalizado (TNF) según la Norma AATCC 8:2013.

\begin{tabular}{clcccc}
\hline \multirow{2}{*}{ Evaluación } & \multicolumn{2}{c}{ Testigo multifibra } & \multicolumn{4}{c}{ Muestras textiles } \\
\cline { 3 - 6 } & \multicolumn{1}{c}{ DW } & TNF & TF20 & TF40 & TF60 \\
\hline \multirow{5}{*}{ Transferencia de } & Di-acetato & $4-5$ & $4-5$ & $4-5$ & $4-5$ \\
color & Algodón blanqueado & $4-5$ & 4 & 4 & 4 \\
& Poliamida & 4 & $4-5$ & 4 & $4-5$ \\
& Poliéster & $4-5$ & $4-5$ & $4-5$ & $4-5$ \\
& Acrílico & 5 & 5 & $4-5$ & $4-5$ \\
& Lana & $4-5$ & 4 & $4-5$ & 4 \\
\hline Cambio de color & & $4-5$ & $4-5$ & $4-5$ & 3 \\
\hline
\end{tabular}

\section{Solidez al sudor}

La estabilidad no satisfactoria del color a la transpiración o sudor se debe al corrimiento o migración del color o al cambio de color del material teñido. Por otra parte, se puede producir un corrimiento sin ningún cambio aparente de color o viceversa, o puede haber tanto corrimiento como cambio de color.

La solidez al sudor fue determinada con la norma ISO 105/E04:2006, que consiste en coser el tejido textil a una multifibra que luego serán sumergidos por 30 min en soluciones ácidas y alcalinas los cuales simulan el sudor del cuerpo humano. Después son colocadas entre placas de resina acrílica ubicadas en el equipo de Transpiración, posterior a ello son introducidas en la estufa por $4 \mathrm{~h}$ a $37^{\circ} \mathrm{C}$. En la Tabla 7 se presentan los resultados obtenidos de solidez de los tejidos al sudor, donde claramente se muestra que la presencia de las nanopartículas en los textiles funcionalizados no ocasiona transferencia ni cambio de color, es decir presentan una excelente solidez al sudor. 
Tabla 7. Resultados de la evaluación de solidez al sudor para los textiles funcionalizados con suspensiones de diferentes concentraciones y del textil no funcionalizado (TNF) según Norma ISO 105/E04:2006.

\begin{tabular}{clcccc}
\hline \multirow{2}{*}{ Evaluación } & \multicolumn{1}{c}{$\begin{array}{c}\text { Testigo multifibra } \\
\text { DW }\end{array}$} & \multicolumn{4}{c}{ Muestras textiles } \\
\cline { 3 - 6 } & Di-acetato & 5 & 5 & 5 & 5 \\
& Algodón blanqueado & 5 & 5 & 5 & 5 \\
\multirow{3}{*}{ Transferencia de } & Poliamida & 5 & 5 & 5 & 5 \\
color & Poliéster & 5 & 5 & 5 & 5 \\
& Acrílico & 5 & 5 & 5 & 5 \\
& Lana & 5 & 5 & 5 & 5 \\
\hline Cambio de color & & 5 & 5 & 5 & 5 \\
\hline
\end{tabular}

\section{Control de color}

La evaluación del cambio de color producido luego de la funcionalización a las diferentes concentraciones de la suspensión de nanopartículas de $\mathrm{ZnO} 2$ al textil teñido, fue realizada haciendo uso del equipo Datacolor 550 TM, Datacolor Spectrum TM. Para el cálculo de las diferencias de color se hizo uso del programa Datacolor TOOLS. La Tabla 8 muestra los resultados de diferencia de color entre el TNF y los textiles funcionalizados con suspensiones de diferentes concentraciones de nanopartículas de $\mathrm{ZnO} 2$. Todas las muestras textiles fueron analizadas con iluminante luz de día (D65) y con el observador patrón de $10^{\circ}$ normalizado por la Comisión Internacional de Iluminación (CIE del término en francés Commission Internationale de L'Eclairage). Donde DL, Da, Db, DC y DH es la diferencia entre el TNF y el textil funcionalizado del punto de claridad sobre el que se encuentran los matices neutros de gris del blanco al negro, medida de rojo/verde, medida de amarillo/azul, medida de la saturación (opaco, intenso) y la medida del matiz o color, respectivamente. De los valores de diferencia de color, se puede apreciar que a mayor concentración de la suspensión de nanopartículas, aumenta la claridad del textil es decir el textil se ve más blanco.

Tabla 8. Diferencias de color de las muestras textiles funcionalizadas con suspensiones de diferentes concentraciones de nanopartículas.

\begin{tabular}{ccccccc}
\hline Muestras textiles & $\Delta$ E CIELab & DL & Da & Db & DC & DH \\
\hline TF20 & 1.81 & -0.10 & -1.73 & -0.55 & 1.64 & 0.77 \\
TF40 & 2.16 & 1.80 & 0.66 & -0.98 & -0.78 & 0.89 \\
TF60 & 4.01 & 3.09 & 1.88 & -1.73 & -2.08 & 1.49 \\
\hline
\end{tabular}

\section{Análisis microbiológico de los textiles}

Los análisis microbiológicos de las telas se realizaron según la norma AATCC 100:2012. El cual consiste en colocar trozos del tejido textil sobre una placa Petri estéril, luego son impregnados con $1 \mathrm{ml}$ de los microorganismos a ensayar a una concentración de 1,5 x $10^{8}$ unidades formadoras de colonias por mililitro $(\mathrm{ufc} / \mathrm{ml})$. Un trozo de tela es sembrado inmediatamente (tiempo cero) y el otro a las $24 \mathrm{~h}$ de incubación a $37^{\circ} \mathrm{C}$. Después de la impregnación e incubación con la cepa a estudiar, el textil se somete a un lavado con $100 \mathrm{ml}$ 
de agua destilada estéril. Luego el agua de lavado es diluido desde $10^{-1}$ hasta $10^{-5}$, posterior a ello son sembrados en medio Agar Tripticase de Soya e incubados por $24 \mathrm{~h}$ a $37^{\circ} \mathrm{C}$, para finalmente realizar las lecturas correspondientes.

Las Tablas 9 y 10 muestran los resultados obtenidos del conteo bacteriano en ufc/ml para la evaluación microbiológica del textil con Pseudomonas aeruginosa y Escherichia coli, respectivamente. El textil no funcionalizado (TNF) y el textil funcionalizado con una suspensión de concentración de $20 \mathrm{~g} / \mathrm{l}$ de nanopartículas $\mathrm{ZnO} 2$, no presentan porcentaje de reducción bacteriana, es decir los microorganismos se han desarrollado.

Los textiles funcionalizados con suspensiones de concentraciones de 40 y $60 \mathrm{~g} / 1$, muestran actividad antimicrobiana después de $24 \mathrm{~h}$ de contacto frente a las dos cepas de bacterias evaluadas, con una reducción de su crecimiento del 100\%

Tabla 9. Resultados del porcentaje de reducción bacteriana del textil no funcionalizado (TNF) y textiles funcionalizados con diferentes concentraciones de las suspensiones de nanopartículas de $\mathrm{ZnO}_{2}$ para la Pseudomonas aeruginosa (ATCC 10145), según Norma AATCC 100:2012.

\begin{tabular}{cccc}
\hline \multirow{2}{*}{ Muestras textiles } & \multicolumn{2}{c}{ Conteo bacteriano $(\mathrm{ufc} / \mathrm{ml})$} & \multirow{2}{*}{$\begin{array}{c}\text { Porcentaje } \\
\text { reducción }(\%)\end{array}$} \\
\cline { 2 - 3 } & Tiempo cero & Tiempo $24 \mathrm{~h}$ & redución \\
TNF & $560 \times 10$ & $>100,000$ & No hay reducción \\
TF20 & $640 \times 10$ & $>100,000$ & No hay reducción \\
TF40 & $180 \times 10$ & 0 & $100 \%$ \\
TF60 & $280 \times 10$ & 0 & $100 \%$ \\
\hline
\end{tabular}

Tabla 10. Resultados del porcentaje de reducción bacteriana del textil no funcionalizado (TNF) y textiles funcionalizados con diferentes concentraciones de las suspensiones de nanopartículas de $\mathrm{ZnO}_{2}$ para la Escherichia coli (ATCC 25922), según Norma AATCC 100:2012.

\begin{tabular}{cccc}
\hline \multirow{2}{*}{ Muestras textiles } & \multicolumn{2}{c}{ Conteo bacteriano $(\mathrm{ufc} / \mathrm{ml})$} & \multirow{2}{*}{$\begin{array}{c}\text { Porcentaje } \\
\text { reducción }(\%)\end{array}$} \\
\cline { 2 - 3 } & Tiempo cero & Tiempo $24 \mathrm{~h}$ & reducción \\
TNF & $140 \times 10$ & $>100,000$ & No hay reducción \\
TF20 & $1040 \times 10$ & $>100,000$ & No hay reducción \\
TF40 & $1370 \times 10$ & 0 & $100 \%$ \\
TF60 & $350 \times 10$ & 0 & $100 \%$ \\
\hline
\end{tabular}

La figura 6, muestra el conteo bacteriano realizado en las placas Petri para el textil no funcionalizado y los textiles funcionalizados. La pigmentación verdosa es característica del desarrollo bacteriano de Pseudomonas aeruginosa que se aprecia claramente en el TNF y el textil funcionalizado con la suspensión de concentración $20 \mathrm{~g} / \mathrm{l}$. Mientras que para el caso de la Escherichia coli se observa una ligera opacidad para las mismas muestras. Por otro lado para los textiles funcionalizados con suspensiones de mayores concentraciones (40 y $60 \mathrm{~g} / \mathrm{l})$ no se desarrolla crecimiento de las cepas bacterianas. 

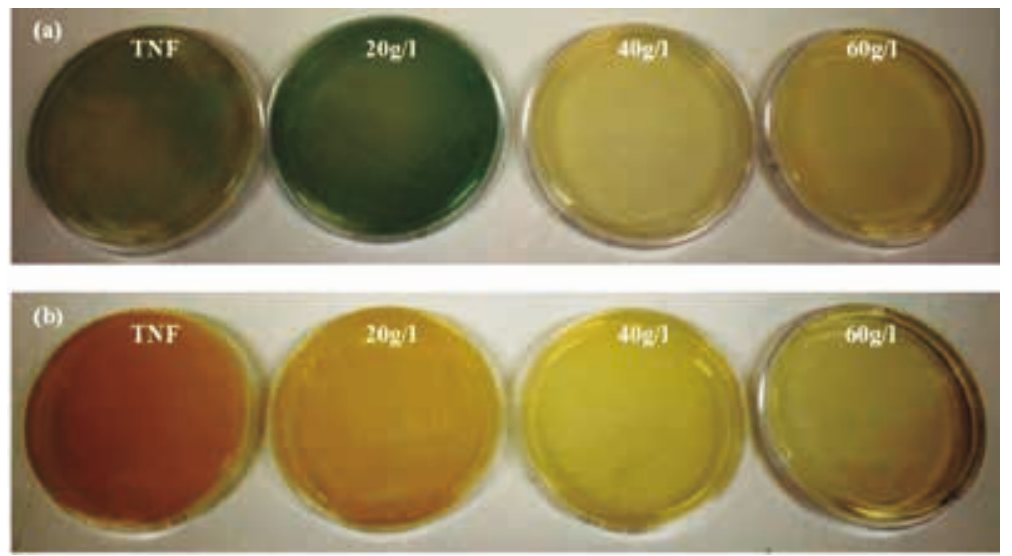

Figura 6. Imágenes de las placas Petri correspondientes al textil no funcionalizado (TNF) y a los textiles funcionalizados a las concentraciones de suspensiones de nanopartículas de $\mathrm{ZnO}_{2}$ indicadas. (a) Corresponde a las placas analizadas para Pseudonomas aeruginosa y (b) Escherichia coli.

\section{Morfología del tejido textil}

La información de la ubicación y distribución de las nanopartículas de $\mathrm{ZnO}_{2}$ en la superficie de las fibras textiles es de gran importancia para entender el comportamiento antimicrobiano que estas presentan, por ello su morfología fue estudiada por microscopía electrónica de barrido empleando un microscopio FEI Quanta 200 operado a $30 \mathrm{kV}$ en el modo imagen.

La figura 7, presenta las micrografías electrónicas de barrido para las muestras funcionalizadas con diferentes concentraciones de la suspensión de $\mathrm{ZnO}_{2}$, (a) 20g/l y (b) $40 \mathrm{~g} / \mathrm{l}$. Las nanopartículas de $\mathrm{ZnO}_{2}$ se encuentran distribuidas en la superficie de la fibra. En el caso del textil obtenido con una suspensión de concentración $20 \mathrm{~g} / \mathrm{l}$, se observa una distribución uniforme, mientras que para el textil funcionalizado con una concentración dos veces mayor, se aprecian también conglomerados de las nanopartículas.
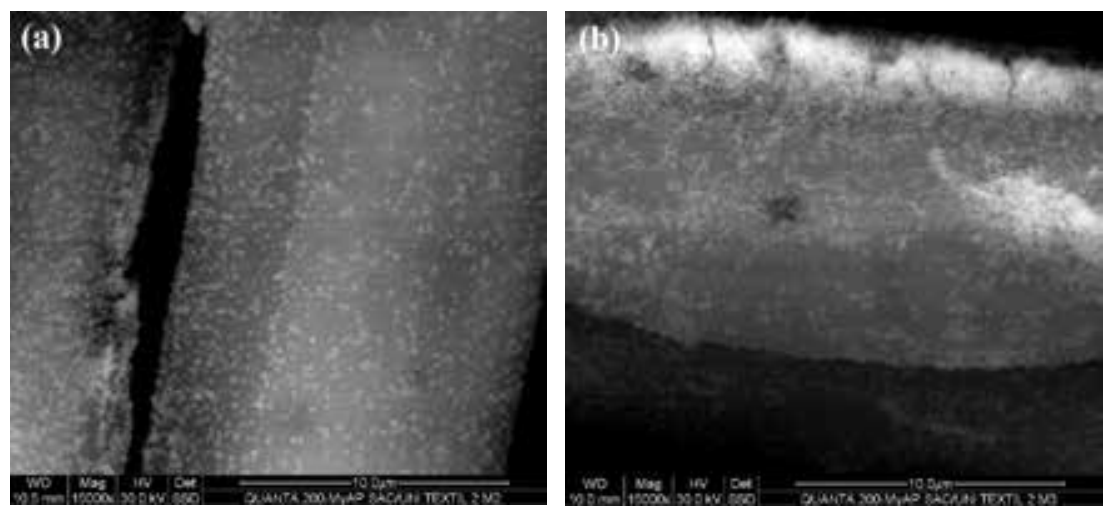

Figura 7. Micrografías electrónicas de barrido para el textil funcionalizado con una suspensión de nanopartículas de $\mathrm{ZnO}_{2}$ de (a) $20 \mathrm{~g} / \mathrm{l}$ y (b) $40 \mathrm{~g} / 1$. 


\section{CONCLUSIONES}

Las nanopartículas de $\mathrm{ZnO}_{2}$ fueron sintetizadas por el método de sol-gel empleando radiación UV durante su síntesis. Por DRX y FTIR se comprobó la presencia de $\mathrm{ZnO}_{2}$ el cual presentó dominios cristalinos de $\sim 4 \mathrm{~nm}$, que se presentó en forma de conglomerados esféricos de tamaños que variaban entre $\sim 80$ a $200 \mathrm{~nm}$.

El textil funcionalizado con nanopartículas de $\mathrm{ZnO}_{2}$ presentó buenas solideces al lavado doméstico, frote y sudor, además se incrementaron los valores de las propiedades físicas, también se originó un cambio de color sobre el textil original pero este cambio de color era previsible y es usual en todas las aplicaciones de acabados textiles en la industria previéndose el mismo en el desarrollo del color. A la concentración de $20 \mathrm{~g} / \mathrm{l}$ de suspensión de nanopartículas de $\mathrm{ZnO}_{2}$ no hubo reducción del crecimiento de las bacterias Pseudonomas aeruginosa y Escherichia coli, mientras que para las suspensiones de concentraciones mayores a $40 \mathrm{~g} / 1$ se redujero al $100 \%$ el crecimiento de bacterias de las cepas indicadas.

\section{AGRADECIMIENTO}

Los autores agradecen a la Dra. Gladys Ocharan y al Dr. Francisco Paraguay por los análisis de microscopía electrónica de barrido. Dos de nosotros (L.E.R.M. y M.M.G.L.) agradecen al Instituto general de Investigación de la Universidad Nacional de Ingeniería (IGI-UNI) por la beca otorgada.

El presente trabajo fue desarrollado bajo la financiación recibida por el Instituto General de Investigación de la Universidad Nacional de Ingeniería (IGI-UNI) y el Programa Nacional de Innovación para la Competitividad y Productividad del Ministerio de Producción del Perú (PNICP).

\section{REFERENCIAS BIBLIOGRÁFICAS}

1. Jocic D. Functional finishing of textiles with responsive polymeric systems. In Jocic D. Surface modification systems for creating stimuli responsiveness of textiles. Enschede: University of Twente; 2010; 37-59.

2. Baurley S. Interactive and experiential desing in smart textile products and applications. Pers Ubiquit Comput. 2004; 8: 274-281.

3. Horrocks AR, Anand SC. Handbook of technical textiles. Cambridge: Woodhear Publishing Ltd.; 2000. p 2-23.

4. Kiekens P, Van der Burght E, Kny E, Uyar T, Milašius R. Functional textiles - from research and development to innovations and industrial uptake. Autex Res J. 2014; 14(4): 219-226.

5. Van Langenhove L, Hertleer C. Smart clothing: a new life. Int J Cloth Sci Tech. 2004; 16(1-2): 63-72. 
6. Pan N, Sun G. Functional textiles for improved performance, protection and health. Cambridge: Woodhead Publishing Ltd.; 2011.p. 360-396.

7. Horrocks AR. Flame retardant challenges for textiles and fibres: New chemistry versus innovatory solutions. Polym. Degrad Stabil. 2011; 96(3): 377-392.

8. Mohsin M, Ahmad SW, Khatri A, Zahid B. Performance enhancement of fire retardant finish with environment friendly bio cross-linker for cotton. J Clean Prod. 2013; 51: 191-195.

9. Scott RA. Textiles for protection. Cambridge: Woodhead Publishing Ltd.; 2005. P. 151215.

10. Uribe C, Maurtua D, Roca E, Brañez M, Román LE, Alvarez J, et al. Obtención de textiles bactericidas. Mundo Textil: Asociación Peruana de Técnicos Textiles. 2014; 127: 26-32.

11. Rajendran S. Advanced textiles for wound care. Cambridge: Woodhead Publishing Ltd.; 2009. p.153-195.

12. Schindler WD, Hauser PJ. Chemical finishing of textiles. Cambridge: Woodhead Publishing Ltd.; 2004. p. 165-174.

13. Bobbarala V. Antimicrobial agents. Rijeka: InTech; 2012. p. 387-406.

14. Román Mendoza LE. Estudio experimental del acabado textil antimicrobiano textil con nanopartículas: obtención, aplicación y control. [Tesis para optar el título de profesional]. Lima: Universidad Nacional de Ingenieria; 2014.

15. Van Langenhove L. Smart textiles for medicine and healthcare: Materials, systems and applications. Cambridge: Woodhead Publishing Ltd.; 2007. p. 26-147.

16. Bartels VT. Physiological comfort of biofunctional textiles. Curr Probl Dermatol. 2006; 33:51-66.

17. Tao X. Smart fibres, fabrics and clothing. Cambridge: Woodhead Publishing Ltd.; 2001. p. 174-197.

18. Abraham GA, Caracciolo PC, Miró Specos M, Escobar G, Hermida L. Nanotecnología para textiles funcionales. Rev SAM. 2012; 1: 3-46.

19. White WC, Monticello RA. Antimicrobial performance of medical textiles. AEGIS Environ. 2002: 1-14.

20. Dastjerdi R, Montazer M, Shahsavan S. A new method to stabilize nanoparticles on textile surfaces. Colloids Surf A. 2009; 345(1-3): 202-210.

21. Zhang F, Wu X, Chen Y, Lin H. Application of silver nanoparticles to cotton fabric as an antibacterial textile finish. Fiber Polym. 2009; 10(4): 496-501.

22. Cioffi N, Torsi L, Ditaranto N, Tantillo G, Ghibelli L, Sabbatini L, et al. Copper nanoparticle/polymer composites with antifungal and bacteriostatic properties. Chem Mater. 2005; 17(21): 5255-5262.

23. Ahmad T, Wani IA, Manzoor N, Ahmed J, Asiri AM. Biosynthesis, structural characterization and antimicrobial activity of gold and silver nanoparticles. Colloids Surf B. 2013; 107: 227-234.

24. Çakir BA, Budama L, Topel Ö, Hoda N. Synthesis of ZnO nanoparticles using PSb-PAA reverse micelle cores for UV protective, self-cleaning and antibacterial textile applications. Colloids Surf A. 2012; 414: 132-139.

25. Gordon T, Perlstein B, Houbara O, Felner I, Banin E, Margel S. Synthesis and 
characterization of zinc/iron oxide composite nanoparticles and their antibacterial properties. Colloids Surf A. 2011; 374(1-3): 1-8.

26. Stevanovic M, Bracko I, Milenkovic M, Filipovic N, Nunic J, Filipic M, et al. Multifunctional PLGA particles containing poly(l-glutamic acid)-capped silver nanoparticles and ascorbic acid with simultaneous antioxidative and prolonged antimicrobial activity. Acta Biomater. 2014; 10(1): 151-162.

27. Makumire S, Chakravadhanula V, Köllisch G, Redel E, Shonhai A. Immunomodulatory activity of zinc peroxide $(\mathrm{ZnO} 2)$ and titanium dioxide (TiO2) nanoparticles and their effects on DNA and protein integrity. Toxicol Lett. 2014; 227(1): 56-64.

28. Colonia R, Solís JL, Gómez M. Bactericidal, structural and morphological properties of $\mathrm{ZnO} 2$ nanoparticles synthesized under UV or ultrasound irradiation. Adv Nat Sci: Nanosci Nanotechnol. 2014; 5(1): 015008.

29. Meleney FL. Zinc Peroxide in Surgical Infections. Am J Nurs. 1941; 41(6): 645-649.

30. Meleney FL, Harvey HD. The Combined use of zinc peroxide and sulfanilamide in the treatmnet of chronic, undermining, burrowing ulcers due to the micro-Aerophilic Hemolytic Streptococcus. Ann Surg. 1939: 1067-1094.

31. Sunderland D, Binkley S. The use of zinc peroxide in infected tumors and radiation necrosis. Case Memorial hospital. 1939; 35(5): 606-616.

32. Johnson BA, Meleney FL. The antiseptic and detoxifying action of zinc peroxido on certain surgical aerobic, anaerobic and micro-aerophilic bacteria. Ann Surg. 1939; 109(6): 881-912.

33. Brinker CJ, Scherer GW. Sol-Gel Science: The Physics and Chemistry of Sol-Gel Processing. London: Academic Press Limited;1990.

34. Escobedo-Morales A, Esparza R, García-Ruiz A, Aguilar A, Rubio-Rosas E, Pérez R. Structural and vibrational properties of hydrothermally grown $\mathrm{ZnO} 2$ nanoparticles. J Cryst Growth. 2011; 316: 37-41.

35. Han X, Liu R, Chen W, Xu Z. Properties of nanocrystalline zinc oxide thin films prepared by thermal decomposition of electrodeposited zinc peroxide. Thin Solid Films. 2008; 516: 4025-4029.

36. Cullity BD. Elements of X-ray diffraction. Massachusetts: Addison-Wesley Publishing Company Inc.; 1956. p. 149-173.

37. Drmosh QA, Gondal MA, Yamani ZH, Saleh TA. Spectroscopic characterization approach to study surfactants effect on $\mathrm{ZnO} 2$ nanoparticles synthesis by laser ablation process. Appl Surf Sci. 2010; 256: 4661-4666.

38. Ibarra L, Marcos-Fernández A, Alzorriz M. Mechanistic approach to the curing of carboxylated nitrile rubber (XNBR) by zinc peroxide/zinc oxide. Polymer. 2002; 43: 1649-1655.

39. Gondal MA, Drmosh QA, Yamani ZH, Saleh TA. Synthesis of $\mathrm{ZnO} 2$ nanoparticles by laser ablation in liquid and their anneling transformation into $\mathrm{ZnO}$ nanoparticles. Appl Surf Sci. 2009; 256: 298-304.

40. Cheng S, Yan D, Chen JT, Zhuo RF, Feng JJ, Li HJ, et al. Soft-template synthesis and characterization of $\mathrm{ZnO} 2$ and $\mathrm{ZnO}$ hollow spheres. J Phys Chem C. 2009; 113(31): 13630-13635.

41. Saville BP. Physical testing of textiles. Cambridge: Woodhead Publishing Ltd.; 2000. p. 77-114. 